\title{
Situación de los Sistemas de Información Territorial para la gestión municipal: caso de la GAM, Costa Rica, 2018
}

\author{
Situation of Territorial Information Systems usage for \\ municipal management: case of the Greater Metropolitan Area \\ (GMA), Costa Rica, 2018
}

\author{
Douglas Guillen-Montero ${ }^{1}$ \\ Municipalidad de San José, Costa Rica \\ Oscar Antonio Núñez-Román ${ }^{2}$ \\ Municipalidad de San José, Costa Rica \\ Jacqueline Vargas-Bogantes ${ }^{3}$ \\ Municipalidad de San José, Costa Rica \\ Luis Mauricio Vega-Ramírez ${ }^{4}$ \\ Municipalidad de San José, Costa Rica.
}

\begin{abstract}
Resumen
Cuando se habla de un Sistema de Información Geográfica -SIG- Municipal es básicamente el mismo principio de un SIG Corporativo, es decir, un sistema que se ha diseñado para cumplir múltiples objetivos de diversos departamentos de una misma organización. Para que un SIG Municipal sea exitoso es importante, que la organización planifique los alcances que tendrá con la implementación de dicha herramienta y los resultados esperados, a partir de objetivos reales. Este artículo muestra
\end{abstract}

1 Geógrafo \& cartógrafo, Departamento de Información Catastral y Geográfica, Municipalidad de San José. Correo electrónico: dguillen09@gmail.com (Dhttps://orcid.org/0000-0003-2880-461X 2Geógrafo, Oficina Municipal de Gestión del Riesgo a Desastres, Municipalidad de San José. Docente, Universidad Católica de Costa Rica. Correo electrónico: oscarnuz1986@gmail.com (D) https://orcid.org/0000-0001-5583-1053

3 Geógrafa, Dirección de Desarrollo Urbano, Municipalidad de San José.

Correo electrónico: jakyvargasb@gmail.com (D) https://orcid.org/0000-0002-2079-1618

4 Geógrafo, Dirección de Desarrollo Urbano, Municipalidad de San José.

Correo electrónico: lumavera@gmail.com (iD https://orcid.org/0000-0002-4655-7029 
la situación en la que se encuentran los 31 municipios de la Gran Área Metropolitana de Costa Rica, en cuanto al desarrollo de los Sistemas de Información Territorial -SIT- para la gestión de los diferentes procesos municipales y de qué manera, se ha logrado o no vincular la información no solamente geográfica sino de diversa índole de interés institucional dentro del SIG Municipal, de acuerdo con el sondeo realizado entre los meses de mayo a octubre del 2018. Para este estudio, se considera la delimitación geográfica establecida por el Plan GAM 82, el cual a pesar de que cuenta con propuestas posteriores para su reforma, no han sido oficializadas. Dicho Plan, se mantiene como documento de consulta e incluso figura como instrumento de ordenamiento territorial en aquellos municipios, que no cuentan con plan regulador o cuentan con uno parcialmente aprobado.

Palabras clave: SIG, SIT, Municipalidad, Plan Regulador, Ordenamiento Territorial, GAM, Plan GAM 82.

\begin{abstract}
When referring to Municipal Geographic Information Systems (GIS) the underlying principle remains unaltered as that in Corporate GIS, namely: a system that has been designed to meet multiple objectives within different departments of the same organization. For M-GISs to be successful it is important for the organization to plan in advance the scope and expected results sought by their implementation based on realistic objectives. The present article exposes the current status prevailing in the 31 municipalities that comprise the Greater Metropolitan Area of Costa Rica (locally known as Gran Area Metropolitana (GAM)), regarding the degree of progress in the development of Territorial Information Systems (TIS) as management tools for the execution of different municipal processes and how it has been possible, or not, to feed institutional relevant information, of geographical and non-geographical nature, into M-GISs; the presented situational data stems from a survey carried out between May and October, 2018. For this study, the geographical delimitation is that established in the Regional Urban Development Plan of 1982 (Plan GAM 82) even though it is fully recognized that it has undergone multiple unofficial modifications and reform proposals since its enactment in 1982. Plan GAM 82 remains as a consultation document and is even utilized as an instrument for territorial planning by municipalities that lack or possess a partially approved territorial regulatory plan.
\end{abstract}

Keywords: M-GIS; GIS; TIS; Municipality; Territorial Regulatory Plan; Territorial Planning; Greater Metropolitan Area (GMA; GAM in Spanish); Plan GAM 82.

\title{
Introducción
}

Los países de la región Centroamericana presentan un importante núcleo de concentración, en cuanto a las principales actividades económicas, sociales, culturales e institucionales. En el caso de Costa Rica, está compuesta por las cuatro ciudades principales San José, Heredia, Cartago y Alajuela que se subdividen en 31 cantones para formar lo que se conoce como la Gran Área Metropolitana -GAM-. La GAM, cuenta con una superficie de $2044 \mathrm{~km}^{2}$ aproximadamente y se estima que la población 
Douglas Guillen-Montero, Oscar Antonio Núñez-Román, Jacqueline Vargas-Bogantes,

Luis Mauricio Vega-Ramirez

Situación de los Sistemas de Información Territorial para la gestión municipal:

caso de la GAM, Costa Rica, 2018

proyectada para el año 2020, será del 53\% (2.268.248 Ha) del total del país, según el Instituto Nacional de Estadística y Censo (INEC, 2011).

La GAM se fue constituyendo a partir de los años 70's por el alto crecimiento de población, a raíz de este evento se provoca la conurbación de las principales ciudades urbanas del país (Cartago, San José, Heredia y Alajuela), lo cual llevó a un crecimiento acelerado y desorganizado. Esta tendencia de crecimiento conlleva "a un desequilibrio en la ubicación de actividades, como la concentración de comercios, servicios y vivienda en lugares no aptos para la localización de áreas residenciales" (POTGAM 2011-2030. p.3).

Para tratar de controlar dicho crecimiento, el Instituto Nacional de Vivienda y Urbanismo -INVU-, bajo las disposiciones de la Ley de Planificación Urbana 4240 crea el "Plan Regional de Desarrollo Urbano del Gran Área Metropolitana" conocido como "el plan GAM 82", que delimita y establece las normas para regular el correcto desarrollo urbano en esa área" (Gaceta 119- 1982).

Una de las herramientas, que ayudan a poner en marcha algunas estrategias a ejecutar en los instrumentos de planificación local, son los Sistemas de Información Geográfica (SIG). Estos se utilizan para el procesamiento y almacenamiento de información, que se deriva del espacio geográfico. Tal como lo expresa Tomlinson (2007), un SIG consta de diferentes elementos como: Datos (puntos, líneas, polígonos), Software (Comercial o Libre) y Equipo para el almacenamiento, procesamiento y análisis de los datos, la cual se encuentra representada gráficamente por medio de mapas, esquemas o modelajes de información.

Por otra parte, cuando la información territorial es almacenada y procesada, se está creando un Sistema de Información Territorial (SIT). Según Villalobos (2009), cuando el alcance de un SIG se asocia a la jurisprudencia de un Gobierno, se transforma entonces en un SIT.

La tecnología de los SIG constituye en este sentido una de las herramientas adecuadas de manejo de información, ya que, al usar el modelo de base de datos georreferenciados, se asocia a un conjunto de información gráfica en forma de planos o mapas a bases de datos espaciales o convencionales. Esto, sintéticamente quiere decir, que los SIG tienen como característica principal que el manejo de la información gráfica y alfanumérica 
se realiza de forma integrada pudiendo abordar de este modo, aspectos de alta complejidad relacionada en el tema planteado.

Es importante, disponer de la estructura necesaria para la construcción, actualización y operación integral de bases de datos y viabilidad de la información, tendiendo a su manejo en tiempo real, para tender a la construcción automática y veloz de información. Indudablemente, la tecnología SIG permite solucionar amplias necesidades técnicas y al mismo tiempo, su uso ha impulsado una modificación estructural del accionar teórico/práctico, en el planeamiento de estas soluciones.

A pesar de que las municipalidades actualmente están invirtiendo más en tecnologías de información, y cuentan con mayor recurso humano y económico que en años anteriores, aun se deja de entre ver, una poca integración de sus bases de datos interdepartamentales, que propicie la creación de un sistema de información territorial para gestión municipal.

Esto puede estar relacionado con la ausencia de conocimiento, por parte de algunas autoridades y técnicos, en cuanto al potencial que poseen los SIT para mejorar la gestión de datos asociados al territorio. Existen otros factores, como la voluntad política, la falta de coordinación a lo interno de la estructura organizacional de los gobiernos locales y el estado de actualización de la base de datos municipal, que inciden en la dificultad de maximizar los beneficios de tener un SIT exitoso y eficiente.

No obstante, con este estudio se pretende comprobar el impacto que han tenido las municipalidades de la GAM a partir de la implementación de un SIT dentro de la organización y como ha incidido el cambio tecnológico en sus procesos de gestión, a partir de las dinámicas y exigencias actuales relacionadas con la digitalización de la información, la transformación hacia ciudades inteligentes e interconectadas y el involucramiento de los habitantes a partir de los datos abiertos.

Por lo tanto, el objetivo general de la presente investigación es determinar un índice sobre el nivel de desarrollo SIT, para la comparación entre los 31 municipios de la GAM. Así mismo, fue necesario establecer un marco de indicadores relacionados con los procesos de administración territorial y, además, construir una metodología para el cálculo del índice en cuestión.

Para tal efecto, se recopilaron los datos a partir del censo aplicado en cada una de las municipalidades, entrevistas con expertos y visitas de 
Douglas Guillen-Montero, Oscar Antonio Núñez-Román, Jacqueline Vargas-Bogantes,

Luis Mauricio Vega-Ramirez

Situación de los Sistemas de Información Territorial para la gestión municipal:

caso de la GAM, Costa Rica, 2018

campo, que enfocaran el análisis de la información a nivel de la gestión local, desarrollos tecnológicos y la planificación para la toma de decisiones.

Mapa 1. Área de estudio: Gran Área Metropolitana de Costa Rica, 2018.

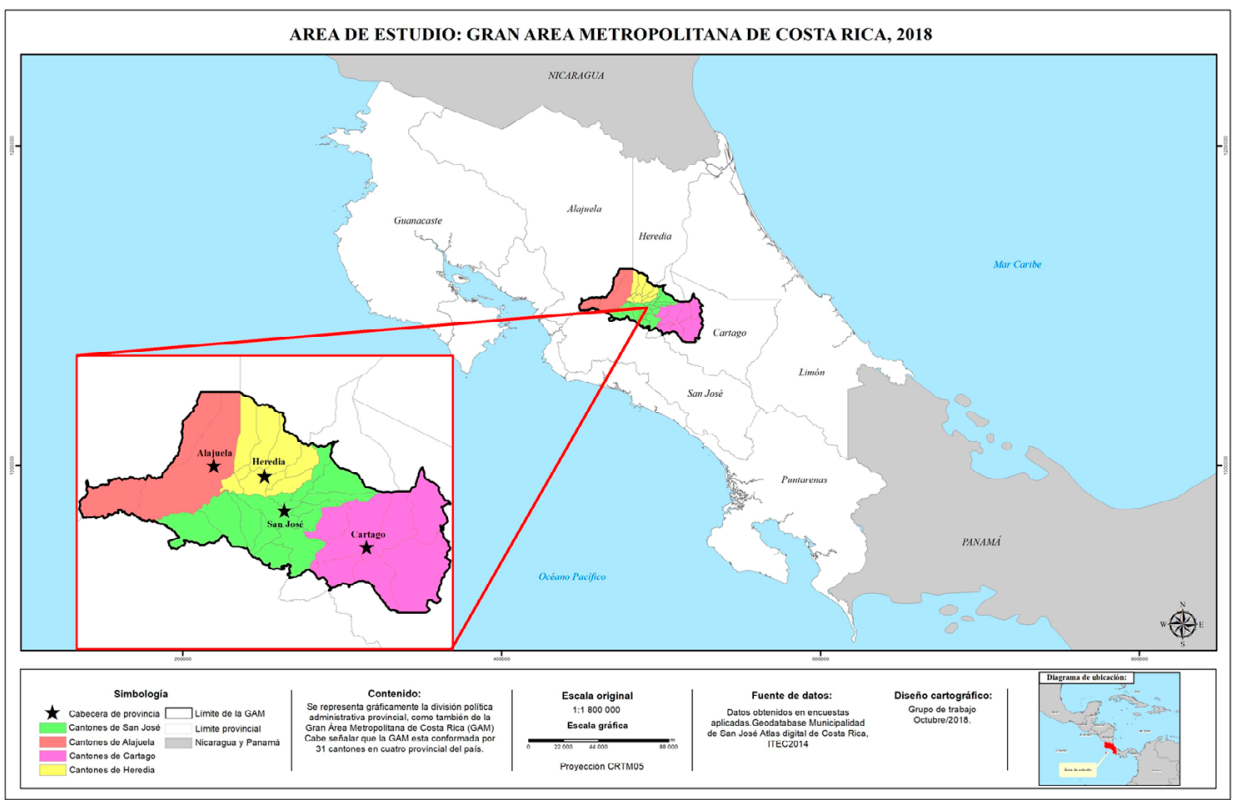

\section{Metodología}

Para el desarrollo de la investigación y la recolección de la información de campo, se utilizaron diferentes mecanismos e instrumentos de trabajo, así como también la codificación de un cuestionario medido a través de un índice, que permite lograr un análisis comparativo entre las 31 municipalidad de estudio.

\section{Recolección de información de fuentes primarias:}

Los insumos de información para este estudio serán descritos en los siguientes apartados según el tipo de consulta realizada, tal como se detalla a continuación:

Censo y visitas a los municipios:

Se diseña un cuestionario que consta de 25 preguntas abiertas y cerradas, estructurada con la finalidad de recolectar datos e información sobre la gestión, que realiza en diferentes ámbitos y el control urbano aplicado. 
Se combina una serie de estrategias para recolectar la información, el 95\% del censo se realiza de manera presencial en los municipios de estudio, y el restante 5\% se realiza vía telefónica y correo electrónico. Así mismo, se documenta mediante un archivo fotográfico algunos aspectos relevantes de las municipalidades en cuanto a equipos utilizados, sitios de interés y procesos. Dichas visitas se llevaron a cabo entre los meses de mayo a noviembre del año 2018. (Ver fotografías de Anexo 1 y 2)

\section{Población entrevistada:}

La población entrevista, se trata de aquellos funcionarios que tenían a su cargo la coordinación en el departamento de Información Catastral, Sistema de Información Institucional o bien, que formarán parte del grupo de administradores SIG, en cada una de las municipalidades.

Algunos otros datos que se requerían consultar en el cuestionario fueron completados con funcionarios de los departamentos de Plataforma de Servicios, encargados del Plan Regulador, Recursos Humanos y Estadística.

No obstante, se aclara que se aplica un único cuestionario por municipio, teniendo en cuenta que el instrumento abarca diferentes aspectos de la gestión municipal, los cuales, al no estar concentrados en un mismo departamento, requieren la consulta de diferentes funcionarios para completarse.

Entrevista con expertos:

La información recopilada en la primera etapa, permite identificar que existen algunas municipalidades que han logrado resultados favorables, a partir de sus Sistemas de Información Institucionales, por lo que se coordinó la visita a dos municipalidades, específicamente con los encargados y administradores del SIG, para generar un proceso de retroalimentación e intercambio de experiencias y ampliar los hallazgos sobre las ventajas y acciones, que les permiten lograr mayor competitividad según el resto de la muestra.

Unidad de Análisis y tamaño de la muestra:

En relación con la unidad de análisis, se refiere a cada municipio de la GAM de Costa Rica, que comprende 31 cantones divididos en áreas metropolitanas, tal como lo estipula el Plan GAM 82 de la siguiente forma: 
Douglas Guillen-Montero, Oscar Antonio Núñez-Román, Jacqueline Vargas-Bogantes,

Luis Mauricio Vega-Ramirez

Situación de los Sistemas de Información Territorial para la gestión municipal:

caso de la GAM, Costa Rica, 2018

Área metropolitana de San José: San José, Escazú, Desamparados, Aserrí, Goicoechea, Alajuelita, Vásquez de Coronado, Tibás, Moravia, Montes de Oca, Curridabat y las Zona de Control Especial de Mora y Santa Ana.

Área metropolitana de Alajuela: Alajuela, Poás y Atenas.

Área metropolitana de Cartago: Cartago, Paraíso, La Unión, Alvarado, Oreamuno y el Guarco.

Área metropolitana de Heredia: Heredia, Barva, Santo Domingo, Santa Bárbara, San Rafael, San Isidro, Belén, Flores, San Pablo.

\section{Nivel de desarrollo e implementación de los Sistemas de Infor- mación Territorial}

El objetivo en este apartado es analizar comparativamente la situación actual de los SIT de cada municipalidad por medio de un Índice y crear un espacio para retroalimentación, entre los diferentes actores de la gestión local de los 31 cantones de la GAM en temas de innovación tecnológica, gestión municipal y experiencias con relación a dicha temática.

Para comparar el nivel de desarrollo e implementación de los SIT de los municipios, es necesario el criterio del equipo investigador con el propósito de clasificar la información en diferentes dimensiones, a partir de las visitas de campo a cada una de las municipalidades, así como de la retroalimentación recibida en las entrevistas y reuniones, que se desarrollaron con expertos de las Municipalidades de Escazú y Cartago.

Es importante aclarar, que se consideran las experiencias de ambos cantones, en función de sus desarrollos e implementación de tecnologías para la gestión de sus procesos, por lo cual, en este estudio se realiza una visita extra a ambas municipalidades, con la finalidad de conocer a profundidad el proceso y la experiencia adquirida, para alcanzar la implementación de los SIT.

Para este caso particular, se consideraron dos dimensiones y en cada una de ellas una serie de indicadores que se desglosaron de la información censada, tal como se detalla a continuación: 
Douglas Guillen-Montero, Oscar Antonio Núñez-Román, Jacqueline Vargas-Bogantes,

Luis Mauricio Vega-Ramírez

Situation of Territorial Information Systems usage for municipal management: case of the Greater Metropolitan Area (GMA), Costa Rica, 2018

\section{Tabla 1. Indicadores por dimensión de análisis para crear el Índice de Situación de Información Territorial -SIT- en los municipios de la GAM costarricense.}

\begin{tabular}{|c|c|c|}
\hline $\begin{array}{l}\text { Dimensión de } \\
\text { Análisis }\end{array}$ & Indicadores de por dimensión & Descripción del indicador \\
\hline \multirow{11}{*}{$\begin{array}{l}\text { Desarrollo de } \\
\text { SIG y Aplicati- } \\
\text { vos (DSA) }\end{array}$} & Cuentan con un SIG & $\begin{array}{l}\text { Cuentan, no cuentan o están en proceso } \\
\text { de implementar un SIG en la institución }\end{array}$ \\
\hline & Tipo de software que utilizan & $\begin{array}{c}\text { El tipo de SIG que utilizan, Comercial, } \\
\text { Libre o Ambos softwares }\end{array}$ \\
\hline & $\begin{array}{l}\text { Cuentan con un departamento } \\
\text { de SIG }\end{array}$ & $\begin{array}{l}\text { Cuentan, no cuentan o están en proceso } \\
\text { de crear un departamento de SIG }\end{array}$ \\
\hline & $\begin{array}{l}\text { Cuentan con un coordinador } \\
\text { del SIG }\end{array}$ & $\begin{array}{l}\text { Cuentan, no cuentan o están en proceso } \\
\text { de asignar un coordinador de SIG }\end{array}$ \\
\hline & $\begin{array}{l}\text { Nivel de vinculación con Tec- } \\
\text { nologías de Información }\end{array}$ & $\begin{array}{l}\text { Cuentan, no cuentan o están en proceso } \\
\text { de tener vinculación con T.I en temas } \\
\text { relacionados al SIG Municipal }\end{array}$ \\
\hline & $\begin{array}{l}\text { Cuentan con un SIG } \\
\text { centralizado }\end{array}$ & $\begin{array}{l}\text { Cuentan, no cuentan o están en proceso } \\
\text { centralizar el SIG Municipal }\end{array}$ \\
\hline & $\begin{array}{l}\text { Cuentan con un SIG } \\
\text { interconectado }\end{array}$ & $\begin{array}{l}\text { Cuentan, no cuentan o están en proceso } \\
\text { centralizar interconectar el SIG Munici- } \\
\text { pal entre sus departamentos usuarios }\end{array}$ \\
\hline & Cuentan con un visor interno & $\begin{array}{l}\text { Cuentan, no cuentan o están en proceso } \\
\text { de construcción del visor interno para } \\
\text { consulta de los funcionarios }\end{array}$ \\
\hline & Cuentan con un visor externo & $\begin{array}{l}\text { Cuentan, no cuentan o están en proceso } \\
\text { de construcción del visor externo para } \\
\text { consulta de la ciudadanía en general }\end{array}$ \\
\hline & $\begin{array}{l}\text { Si tienen desarrollos adicionales } \\
\text { (apps, visores, otros) }\end{array}$ & $\begin{array}{l}\text { Cuentan, no cuentan o están en proceso } \\
\text { de desarrollo tecnológicos vinculados al } \\
\text { SIG Municipal }\end{array}$ \\
\hline & $\begin{array}{l}\text { Tipo de almacenamiento de la } \\
\text { información (servidor, nube, } \\
\text { local) }\end{array}$ & $\begin{array}{l}\text { El tipo de almacenamiento de la infor- } \\
\text { mación que utiliza servidor local, nube } \\
\text { de datos o ambas herramientas. }\end{array}$ \\
\hline
\end{tabular}


Douglas Guillen-Montero, Oscar Antonio Núñez-Román, Jacqueline Vargas-Bogantes,

Luis Mauricio Vega-Ramírez

Situación de los Sistemas de Información Territorial para la gestión municipal:

caso de la GAM, Costa Rica, 2018

\begin{tabular}{|c|c|c|}
\hline \multirow{7}{*}{$\begin{array}{l}\text { Gestión Mu- } \\
\text { nicipal para la } \\
\text { Planificación } \\
\text { (GMP) }\end{array}$} & Cuenta con Plan Regulador & $\begin{array}{l}\text { Cuentan, no cuentan o están en proceso } \\
\text { de desarrollo del Plan Regulador }\end{array}$ \\
\hline & $\begin{array}{c}\text { Cuenta con proyectos de mejora } \\
\text { para la Gestión Municipal }\end{array}$ & $\begin{array}{l}\text { Cuentan, no cuentan o están en proceso } \\
\text { de desarrollo de proyectos de mejora } \\
\text { para la Gestión Municipal }\end{array}$ \\
\hline & $\begin{array}{l}\text { Implementación del SIG en la } \\
\text { Gestión de Riesgos }\end{array}$ & $\begin{array}{c}\text { Cuentan, no cuentan o están en proceso } \\
\text { de implementación del SIG en la gestión } \\
\text { de riesgos (consulta, actualización y } \\
\text { mantenimiento) }\end{array}$ \\
\hline & $\begin{array}{l}\text { Implementación del SIG en la } \\
\text { Gestión de Usos de Suelo }\end{array}$ & $\begin{array}{c}\text { Cuentan, no cuentan o están en proceso } \\
\text { de implementación del SIG en la gestión } \\
\text { de usos de suelo (consulta, actualización } \\
\text { y mantenimiento) }\end{array}$ \\
\hline & $\begin{array}{l}\text { Implementación del SIG en la } \\
\text { Gestión de Visado de Planos }\end{array}$ & $\begin{array}{c}\text { Cuentan, no cuentan o están en proceso } \\
\text { de implementación del SIG en la gestión } \\
\text { de visado de planos (consulta, actualiza- } \\
\text { ción y mantenimiento) }\end{array}$ \\
\hline & $\begin{array}{l}\text { Implementación del SIG } \\
\text { en la Gestión de Permisos } \\
\text { Constructivos }\end{array}$ & $\begin{array}{l}\text { Cuentan, no cuentan o están en proceso } \\
\text { de implementación del SIG en la gestión } \\
\text { de permisos constructivos (consulta, } \\
\text { actualización y mantenimiento) }\end{array}$ \\
\hline & $\begin{array}{l}\text { Implementación del SIG en la } \\
\text { Gestión de Patentes }\end{array}$ & $\begin{array}{c}\text { Cuentan, no cuentan o están en proceso } \\
\text { de implementación del SIG en la gestión } \\
\text { de patentes (consulta, actualización y } \\
\text { mantenimiento) }\end{array}$ \\
\hline
\end{tabular}

Fuente: Elaboración propia, 2019, a partir del censo realizado para el estudio de situación de los Sistemas de Información Territorial de los municipios de la GAM, 2018, Municipalidad de San José.

Debido a que la información de los indicadores que se seleccionaron cuenta con datos cualitativos, se considera como método de estandarización de la información, la clasificación en tres categorías y a cada categoría se le asigna un valor, según su condición tal como se detalla en la tabla 2.

Es importante constatar que para la elaboración de la matriz, lo primero es la asignación del puntaje a cada indicador en los 31 municipios, posteriormente se calcula su valor en porcentaje obtenido por cada categoría, respecto al puntaje más alto que se puede obtener por dimensión, es decir, para este caso en la Dimensión de SIG y Desarrollo de Aplicativos (DSA) al evaluarse 11 indicadores, 33 puntos equivalen al $100 \%$ de su dimensión y para la Dimensión de Gestión Municipal para la Planificación (GMP) 7 indicadores, corresponden a 21 puntos que equivalen al $100 \%$, en 
otras palabras, se sustituyen los puntajes asignados por su equivalente en porcentaje y consecuentemente, cuando se tiene ese Índice de cada dimensión, se calcula el Índice SIT para la Gestión Municipal.

Tabla 2. Costa Rica: Clasificación para estandarización de indicadores y pesos asignados por categoría, para el cálculo de los Índices: DSA, GMP y SIT en los municipios de la GAM, 2019.

\begin{tabular}{|c|c|c|c|c|c|}
\hline $\begin{array}{c}\text { Color de } \\
\text { Represen- } \\
\text { tación en el } \\
\text { mapa }\end{array}$ & Categoría & $\begin{array}{l}\text { Descripción de cada } \\
\text { categoría }\end{array}$ & $\begin{array}{c}\text { Puntaje } \\
\text { asigna- } \\
\text { do por } \\
\text { categoría }\end{array}$ & *Índice por dimensión & $\begin{array}{l}\text { Índice SIT } \\
\text { para la Gestión } \\
\text { Municipal }\end{array}$ \\
\hline & Alta & $\begin{array}{l}\text { El mayor puntaje } \\
\text { indica que cuenta } \\
\text { con el indicador } \\
\text { que se analiza }\end{array}$ & 3 & \multirow{3}{*}{$f=\frac{\left(\mathrm{V}_{1}+\mathrm{V}_{2}+\mathrm{V}_{3}\right) / 100 \%}{\mathrm{~N}_{1}}$} & \multirow{3}{*}{$\begin{array}{c}\boldsymbol{f}=\sum \text { Índice } \\
\text { DSDA+ Índice } \\
\text { DGMP } \\
\mathrm{N}_{2}\end{array}$} \\
\hline & Media & $\begin{array}{l}\text { Un valor interme- } \\
\text { dio indica que se } \\
\text { encuentra en pro- } \\
\text { ceso de desarrollo } \\
\text { o implementación. }\end{array}$ & 2 & & \\
\hline & Baja & $\begin{array}{c}\text { El menor valor } \\
\text { corresponde a la } \\
\text { condición más baja } \\
\text { o nula. }\end{array}$ & 1 & & \\
\hline
\end{tabular}

Fuente: Elaboración propia, 2019, a partir del censo realizado para el estudio de situación de los Sistemas de Información Territorial de los municipios de la GAM, 2018, Municipalidad de San José.

\section{Donde:}

$\mathbf{V}_{1}=$ Porcentaje equivalente de representación de la categoría baja en el municipio para cada dimensión de estudio.

$\mathbf{V}_{2}=$ Porcentaje equivalente de representación de la categoría media en el municipio para cada dimensión de estudio.

$\mathbf{V}_{3}=$ Porcentaje equivalente de representación de la categoría alta en el municipio para cada dimensión de estudio.

$\mathbf{N}_{1}=$ Cantidad total de categoría en las que se clasifican los indicadores.

$\mathbf{N}_{2}=$ Cantidad total de dimensiones de análisis.

* Nota: A cada indicador se le asigna un peso con valores de 1, 2 y 3 según la categoría establecida (Alto, Medio, Bajo), con lo cual se crea una matriz. Dicha matriz requiere que esos valores se recalculen en su equivalente en porcentaje, para lograr generar el índice, y una vez obtenidos los valores $\mathrm{V}_{1}, \mathrm{~V}_{2} \mathrm{y} \mathrm{V}_{3}$ se aplica la formula indicada en la tabla 2 . 
Douglas Guillen-Montero, Oscar Antonio Núñez-Román, Jacqueline Vargas-Bogantes,

Luis Mauricio Vega-Ramirez

Situación de los Sistemas de Información Territorial para la gestión municipal:

caso de la GAM, Costa Rica, 2018

\section{Análisis de los datos}

En cuanto al primer resultado de la DSA, representado en el mapa 2, cabe señalar que el 61\% de los cantones de la GAM, es decir 19 de 31 cantones, poseen dentro de su sistema web visores externos, herramienta que ayuda a la sociedad civil a conocer datos básicos de la distribución territorial del cantón. Entre la información que se expone en muchos de los visores institucionales, tiene relación con la red vial, catastro municipal, cuerpos de agua, puntos de referencia, rutas de recolección, entre otros.

Un dato importante que señalar en esta dimensión es que las municipalidades que se ubican en las primeras posiciones, como Cartago y Escazú, se caracterizan por mantener la vinculación directa entre la implementación del SIG con herramientas, como, por ejemplo: aplicaciones para la recolección de información o datos para el trabajo de campo, así como toma de capturas fotográficas in-situ, aunado a un buen sistema de almacenamiento y respaldo de los datos.

Contar con la información georreferenciada, relacionada a los procesos de gestión de trámites desde su ingreso a la municipalidad hasta la resolución de estos, trae muchas ventajas tanto para la institución como para los ciudadanos, como se pueden mencionar: agilización en los trámites, trazabilidad y transparencia, actualización de los SIT, estadísticas e informes, insumos de valor para la toma de decisiones y planificación del territorio, entre otras. (Ver Foto 1)

Foto 1. App para levantamiento de datos en campo Municipalidad de Barva.

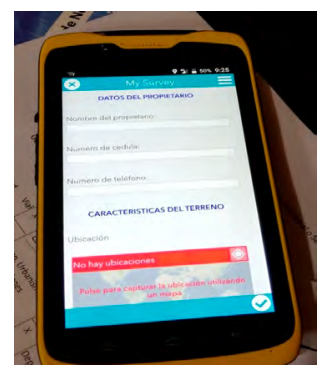

Fuente: Trabajo de campo, 2018. Elaboración propia.

En cuanto a los municipios que se denotan en color rojo, estos 3 municipios corresponden al 10\% de los cantones que, por la falta de 
coordinación entre departamentos para almacenar, compartir y distribuir la información territorial, los coloca en una situación crítica en la GAM. Otro de los aspectos que dificulta la implementación de un SIT actualizado en algunos gobiernos locales, está ligado al estado de su base catastral y registral, ya que no todos se encuentran en zona catastrada, es decir, que sus inconsistencias registrales no se han corregido a través de un proceso de depuración por parte del Registro Nacional.

No obstante, según Lara (2019), el Registro Nacional de la Propiedad, tiene identificado 34 distritos prioritarios, de los cuales 29 son de la provincia de San José y 5 pertenecen a la provincia de Cartago. Cabe señalar que, para el cantón San José los 11 distritos que lo conforman (El Carmen, Merced, Hospital, Catedral, Zapote, San Francisco de Dos Ríos, Uruca, Mata Redonda, Pavas, Hatillo, San Sebastián) están en condición prioritaria.

Dicho panorama, explica una de las principales razones por las cuales el municipio de San José se encuentra en una ubicación intermedia, mientras que el municipio de Cartago se posiciona en el primer lugar de este índice, por contar con zona catastrada oficial, un eficiente aprovechamiento de sus recursos y total prioridad, para la administración de contar con innovación tecnológica.

En cuanto a los resultados de la GMP, cabe señalar que los cantones que están en las primeras posiciones, al igual que en el mapa anterior, ven la importancia de los SIG y su interrelación entre departamentos, secciones o procesos en cuanto a temas como, por ejemplo, permisos de construcción, visados de planos, patentes, plan regulador, gestión del riesgo, entre otros (mapa 3).

Lo anterior, se ve reflejado principalmente en la implementación de visores internos, donde se detalla la georreferenciación y actualización de trámites e información municipal, para ser considerada en la planificación del territorio. (Ver foto 2)

Otros aspectos que respalda a los municipios como Cartago, El Guarco, Escazú, Santa Ana (ver mapa 3), y hace que se encuentren en las primeras posiciones son: contar con un plan regulador aprobado, además de proyectos de la gestión y planificación urbana, entre los cuales se pueden citar: catastro multifinalitario, depuración de bases de datos, 
Douglas Guillen-Montero, Oscar Antonio Núñez-Román, Jacqueline Vargas-Bogantes,

Luis Mauricio Vega-Ramirez

Situación de los Sistemas de Información Territorial para la gestión municipal:

caso de la GAM, Costa Rica, 2018

Mapa 2. Dimensión de desarrollo de SIG y aplicativos en los municipios de la GAM de Costa Rica, 2018.

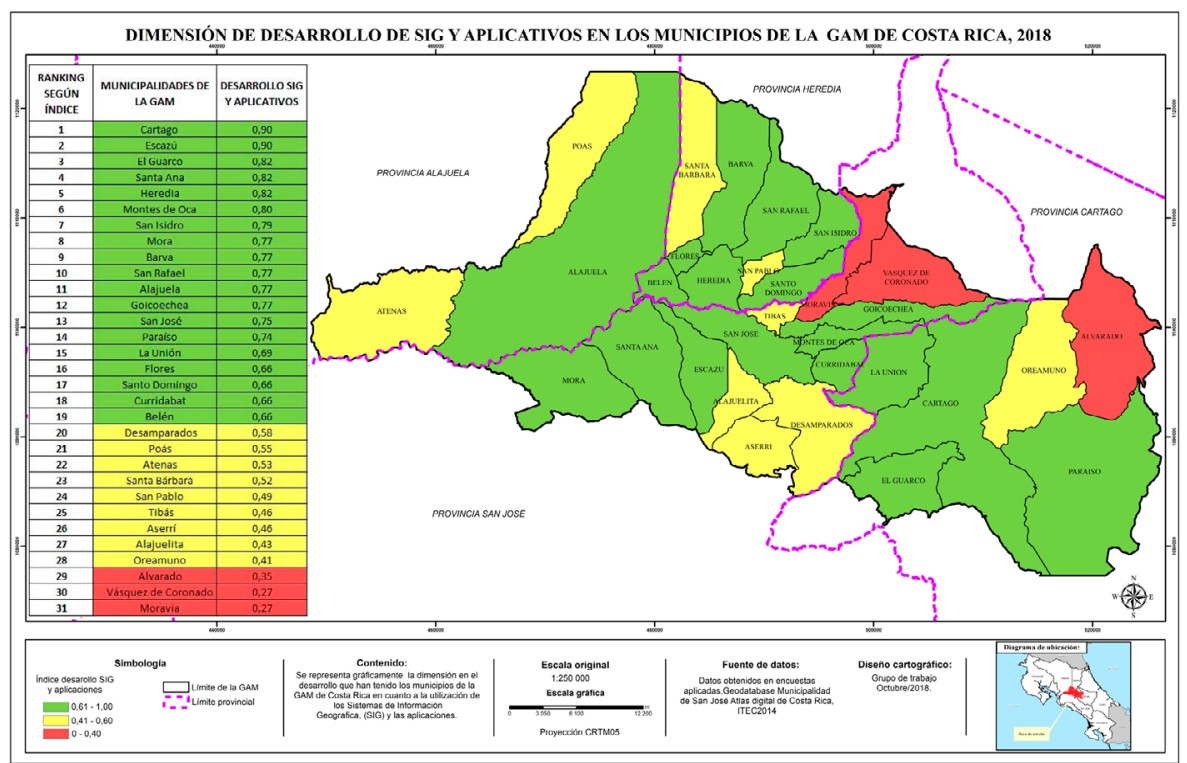

Foto 2. Visor Municipalidad de Alajuela.

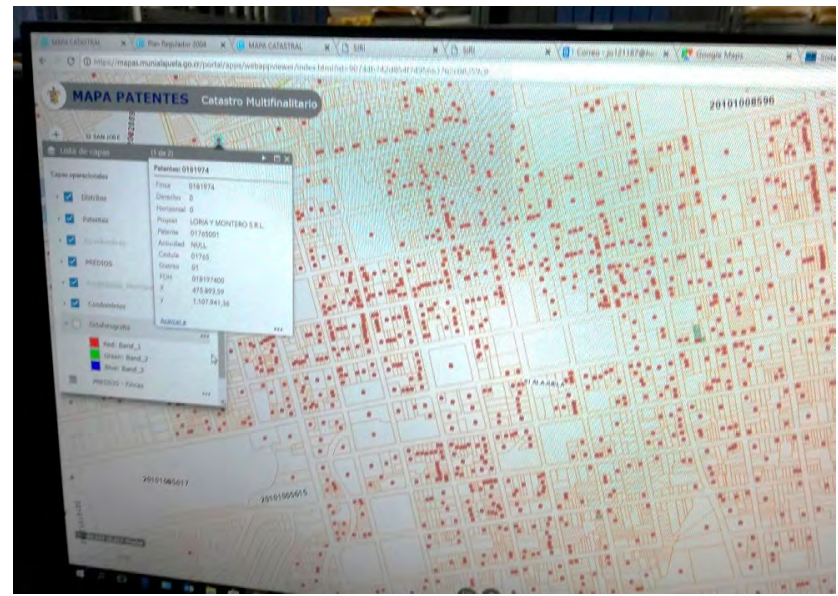

Fuente: Trabajo de campo, 2018. Elaboración propia. 
capacitación a los funcionarios en SIG, facilidad de la divulgación de la información SIT a nivel municipal, entre otros. (Ver foto 3)

Foto 3. Equipo de cómputo Municipalidad de Santa Ana

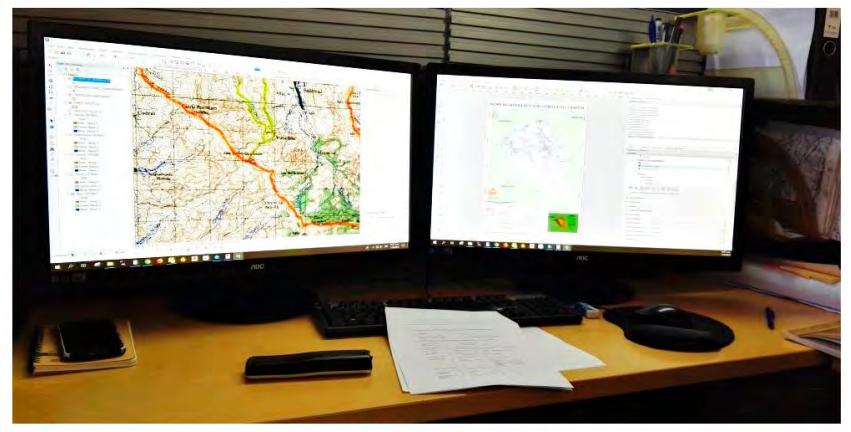

Fuente: Trabajo de campo, 2018. Elaboración propia.

Por otro lado, en cuanto a los municipios que se encuentran rezagados en la dimensión GMP, cabe destacar como primer punto, la falta de un plan regulador, este consiste en un instrumento de planificación y gestión urbana de nivel local, en el que se define la política que orienta a largo plazo, el desarrollo urbano en un determinado territorio. Asimismo, define las acciones estratégicas por ejecutar con relación a la distribución de la población, usos del suelo, vías de circulación, servicios públicos, facilidades comunales, construcción y renovación urbana" (INVU, s.f. p.15).

No obstante, el estudio revela que 11 de los 31 cantones no cuenta con uno o está en su proceso de diseño. Esta limitante en muchos cantones se debe al alto costo económico, las decisiones políticas, también en muchos casos el factor humano, técnico y profesional, la incorporación de la nueva variable ambiental llamada Matriz Hidrogeológica e Índices de Fragilidad Ambiental (IFAS).

Aunado a este tema, es importante hacer referencia a la falta de concordancia entre los planes reguladores de los cantones colindantes, ya que muchas veces la división política-administrativa de un cantón a otro, es un elemento natural o una vía y no existe coherencia entre los reglamentos aplicados a los predios que comparten legislación con otros municipios, de esto surge la importancia de homologación de los usos de suelo, de una manera consensuada entre los gobiernos locales. 
Otro dato para destacar en ellos es el tema de la escasa relación entre las bases de datos de los trámites municipales como: los usos de suelo, patentes y permisos de construcción con la herramienta SIG. Se logra constatar mediante los resultados del censo y entrevistas, la debilidad de no contar con personal capacitado para relacionar, actualizar y administrar dichas bases de datos, con la tecnología de información libre o corporativa.

Mapa 3. Dimensión de gestión municipal para la planificación en los municipios de la GAM de Costa Rica, 2018.

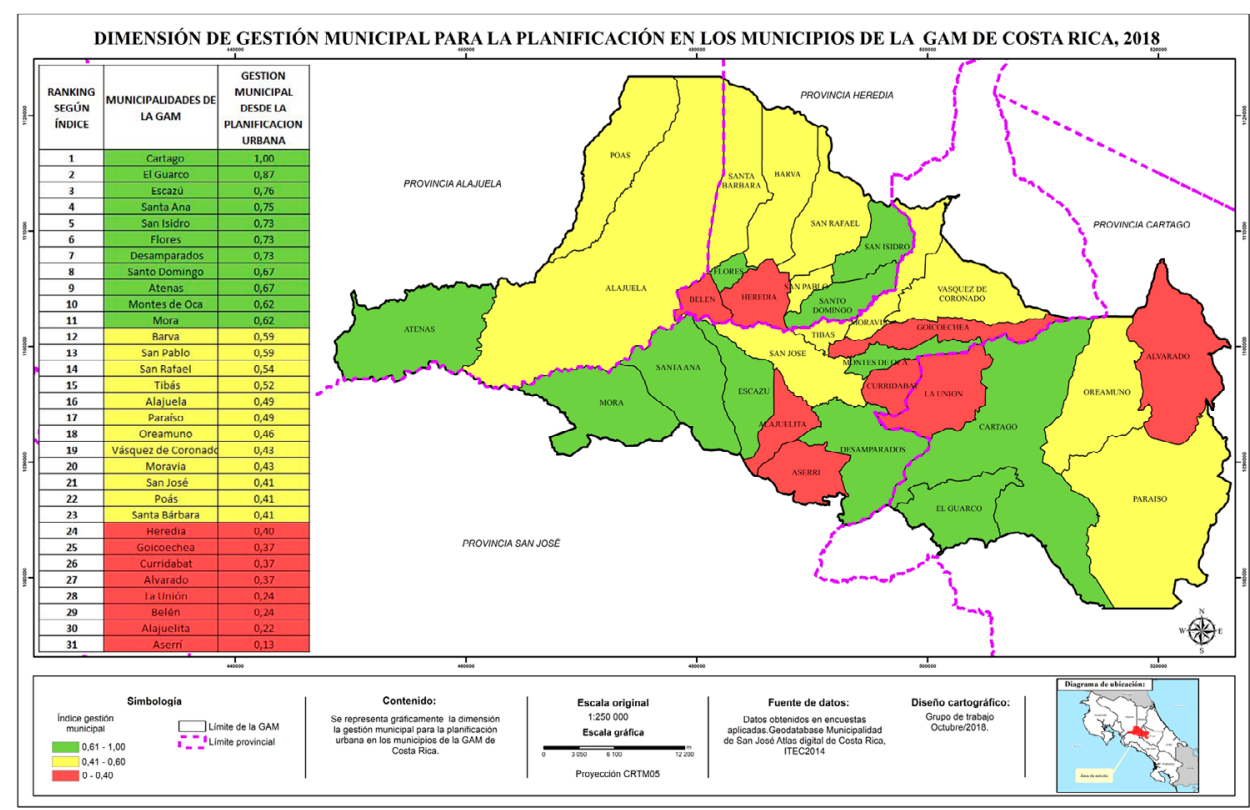

Teniendo en cuenta las dos dimensiones la DSA y GMP, se obtiene como resultado final el Índice SIT para la Gestión Municipal, el cual muestra que los cantones de Cartago, El Guarco y Escazú, han apostado por el desarrollo de forma integral, incorporando sus procesos institucionales como una herramienta de gestión de información, la cual se ha convertido en una aliada para la respuesta inmediata y oportuna a las exigencias de sus contribuyentes (mapa 4).

Con la implementación de este tipo de herramientas tecnológicas y el compromiso que han adquirido los funcionarios de estos municipios, se ha generado un cambio positivo a nivel de sus gestiones, trámites, 
disponibilidad de la información, capacitación e importancia del trabajo en equipo, tal como se visualiza por ejemplo en la foto 4 . Contar con un SIT en los gobiernos locales, ha permitido crear una vinculación entre las diferentes dependencias, que ayuda a la toma de decisiones, control urbano, la planificación y ordenamiento territorial. Así mismo, existen otras municipalidades de la GAM que han creado importantes desarrollos en los SIT, aun cuando no cuentan con un gran presupuesto para operar.

Foto 4. Kiosko Municipal para gestión de trámites en Línea y Unidad de Monitoreo del Acueducto, Municipalidad de Cartago.

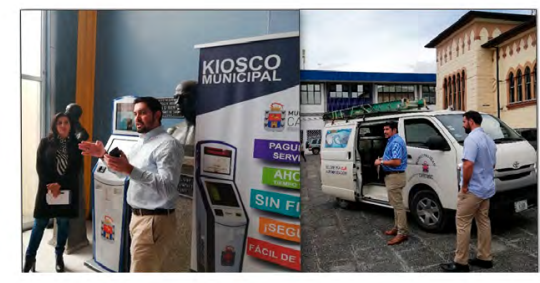

Fuente: Trabajo de campo, 2018. Elaboración propia.

Un dato importante para mencionar es que el cantón de Cartago se encuentra certificado bajo la norma ISO 9001-2015, lo cual le ha permitido fortalecer la estandarización de todos sus procesos, logrando una buena administración pública y territorial, tal como se observa en la foto 5. Según la Oficina de Comunicación y Mercadeo, TEC (2015) el alcalde de cartago indica expresamente que... "existe un planeamiento cuando se dan los permisos de construcciones, y su finalidad es mantener en línea todos los aspectos para no perjudicar ninguna zona de la ciudad”... (Pág.52-53)

En más del 93\% que equivale a 29 municipios, indicaron que cuentan con proyectos de mejora, con el objetivo de buscar mecanismos que coloquen a la vanguardia sus gestiones como gobierno local. Algunos de los que más se repiten son: desarrollo, implementación, vinculación y mantenimiento del SIG corporativo, mejoras institucionales para modernizar el servicio brindado a los contribuyentes, capacitación de su personal y contratación de recurso humano especializado.

Es necesario aclarar, que no en todos los municipios es posible lograr ejecutar dichos proyectos de mejora a corto plazo, debido a su escaso presupuesto, falta de respaldo por parte de la administración local y política, 
Douglas Guillen-Montero, Oscar Antonio Núñez-Román, Jacqueline Vargas-Bogantes,

Luis Mauricio Vega-Ramírez

Situación de los Sistemas de Información Territorial para la gestión municipal:

caso de la GAM, Costa Rica, 2018

Foto 5. Visor Cartográfico (ISO 9001-2015), Municipalidad de Cartago.

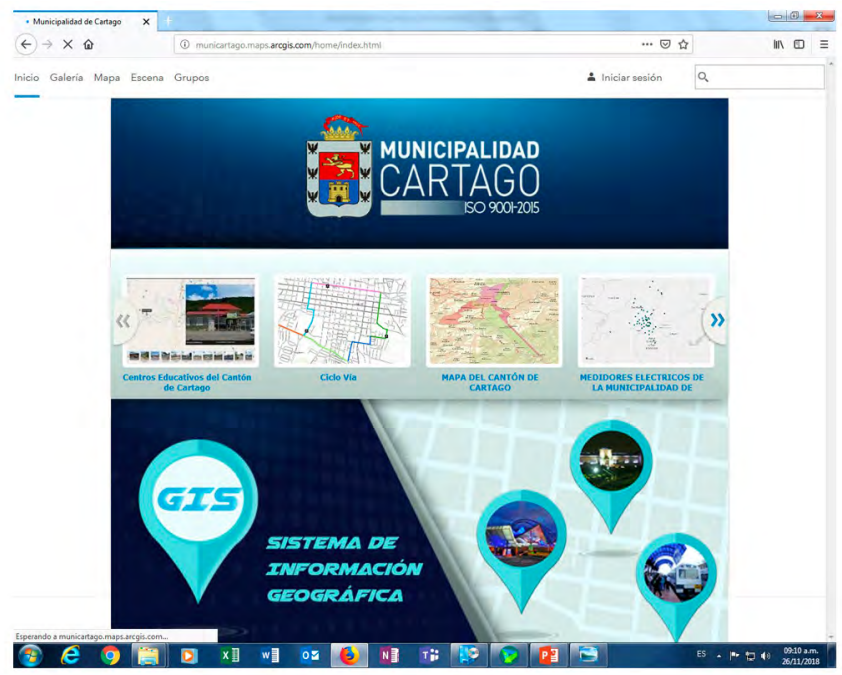

Fuente: Trabajo de campo, 2018. Elaboración propia.

visión e intereses, tal como fue indicado en el cuestionario por parte de los funcionarios de diferentes municipios.

Así, por ejemplo, la municipalidad de Cartago ha establecido alianzas estratégicas con el Ministerio de Ciencia, Tecnología y Telecomunicaciones (MICITT) de manera que sus proyectos converjan para lograr un modelo de ciudad inteligente, sostenible, interconectada, inclusiva y accesible (Oficina de Comunicación y Mercadeo, TEC., 2015). (Ver foto 6).

Foto 6. Centro Comunitario Inteligente de Cartago.

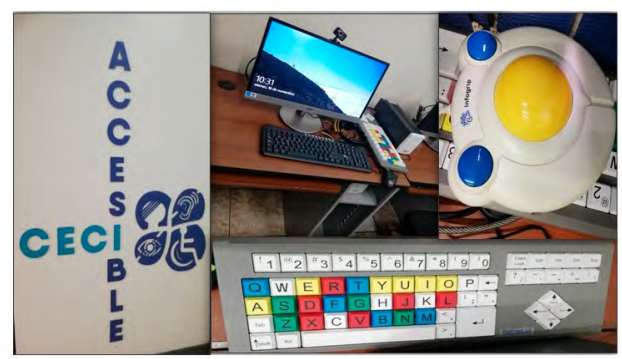

Fuente: Trabajo de campo, 2018. Elaboración propia. 
Para generar mejor gobernanza, el SIT es una herramienta de comunicación que genera transparencia en la institución. Tal como indica Villalobos (2009), "la implementación de un SIT en una institución pública y particularmente en una municipalidad es una tarea difícil", no se puede obviar que la voluntad política juega un papel importante, ya que a través de esta se ponen en marcha los proyectos municipales.

En el caso de los cantones de Aserrí y Alajuelita, son cantones que tienen su plan regulador en proceso, se espera que a partir de su implementación sean cantones que progresen en la adecuada administración del territorio. Tal como se denota en el mapa 4, se trata de cantones con los índices más bajos respecto al resto de cantones de la GAM, con una serie de carencias, dentro de las que más se destaca la falta de voluntad política en atención al desarrollo territorial.

Mapa 4. Índice general de utilización de los SIG para la gestión municipal en la GAM de Costa Rica, 2018.

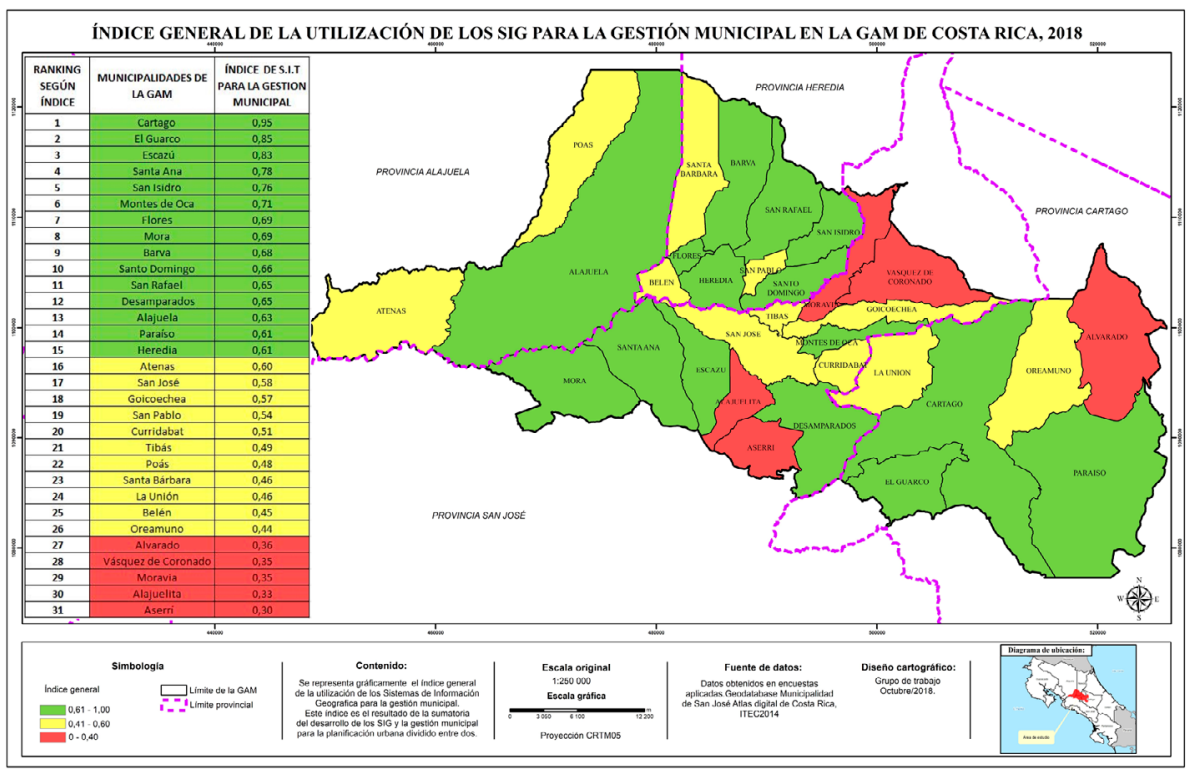


Douglas Guillen-Montero, Oscar Antonio Núñez-Román, Jacqueline Vargas-Bogantes,

Luis Mauricio Vega-Ramírez

Situación de los Sistemas de Información Territorial para la gestión municipal:

caso de la GAM, Costa Rica, 2018

\section{Conclusiones}

El éxito de un SIT corporativo es el resultado de un conjunto de elementos y decisiones, que si se vinculan adecuadamente pueden brindar a los gobiernos locales, una herramienta de gran valor para el desarrollo de sus gestiones diarias y servicios a disposición de sus ciudadanos.

Son muy amplias las opciones de desarrollo, que se pueden lograr a través de los SIT, más aún si se utilizan como una herramienta para la planificación y ordenamiento del territorio, al poner a disposición de sus decisores información actualizada y confiable para realizar sus análisis e interpretaciones, que permitan el desarrollo al cantón que administran.

Como parte de los resultados obtenidos, es posible constatar que las municipalidades que se ubican en las primeras posiciones no son precisamente las que cuentan con el presupuesto más alto pese a esto, han logrado grandes avances, más aún si cuentan con una base catastral limpia y validada por el Catastro Nacional. Caso contrario a lo que ocurre en un municipio tan complejo registral y catastralmente, como es el caso del cantón San José.

El SIT ayuda a optimizar la gestión municipal del territorio, su implementación permite eficiencia en los sistemas de cobros, facilita la planificación urbana, el desarrollo de planes reguladores, así como trazabilidad y transparencia en sus procesos. Lo anterior, se debe complementar con voluntad política, personal capacitada y una visión integradora entre las dependencias, para mejorar la gobernanza.

Es importante, considerar una actualización y oficialización de un instrumento de planificación a nivel regional, que permita a los municipios homologar la información para la gestión efectiva de sus territorios, considerando que en algunas ocasiones los límites políticos-administrativos causan conflictos, entre ellos en la toma de decisiones.

Actualmente, está comprobado a nivel nacional y aún más a nivel internacional, la potencialidad y utilidad que tienen los SIG en el desarrollo científico - técnico y la eficacia de estos, para la gestión del territorio. En Costa Rica, se ha incrementado considerablemente el uso de estos a nivel institucional, tal como se corroboró a través de los resultados obtenidos del censo, donde el 100\% de los municipios entrevistados manifestó contar con un SIG local, también indicaron que se trata de una de sus prioridades en los proyectos de mejora vinculados a este tipo de herramienta. 
Con este tipo de estudio comparativo entre los diferentes gobiernos locales de la GAM, se logró constatar que aun cuando los territorios son muy diferentes en características físicas, socioeconómicas y culturales, es indiscutible que comparten el mismo interés por generar desarrollos tecnológicos, que permitan agilizar sus procesos de gestión y planificación urbana, en pro de lograr ciudades más integrales, inclusivas, sostenibles y accesibles.

\section{Referencias}

Dirección de Urbanismo (INVU). (s.f). Plan de Ordenamiento Territorial de la Gran Área Metropolitana 2011 - 2030, pp. 1 - 146.

Lara, F. (15 de julio de 2019). Mitad de propiedades carecen de limites oficiales. LA NACION. (p. 6)

INEC. (2011). Costa Rica 2020: Población total proyectada al 30 de junio por grupos de edades, según provincia, cantón, distrito y sexo. Recuperado de: http://inec.cr/poblacion/ estimaciones-y-proyecciones-de-poblacion

INVU. (s.f). Manual de Planes Reguladores como Instrumentos de Ordenamiento Territorial, pp. 1-60.

Oficina de Comunicación y Mercadeo, TEC. (abril/junio de 2015). Cartago una ciudad digital. PENSIS, 52-53. Recuperado de: https://repositoriotec.tec.ac.cr/handle/2238/6834?locale-attribute=en

Reglamento 3332. (1982). La Gaceta 119 - 22 de junio de 1982.

Tomlinson, R. (2007). Pensando en el SIG: Planificación del Sistema de Información Geográfica Dirigida a Gerentes. Tercera Edición: ESRI PRESS.

Villalobos, G. (2009). Experiencia Municipal en el Uso e Implementación de un Sistema de Información Territorial. En: Revista Geográfica de América Central 43, 37-47. 